\title{
Role of L-carnitine and Coenzyme Q10 as Adjuvant Therapy in Patients with Type 2 Diabetes Mellitus
}

\author{
Noor Kadhim Mohammed-Jawad ${ }^{1, *}$, May Al- Sabbagh ${ }^{2}$, Kaiss A. AL-Jezaeri ${ }^{3}$ \\ ${ }^{1}$ Department of Clinical Pharmacy, Pharmacy College, Basra University, Basra, Iraq \\ ${ }^{2}$ Department of Clinical Pharmacy, Pharmacy College, Baghdad University, Baghdad, Iraq \\ ${ }^{3}$ Department of medicine, Al-Basra General Hospital \\ *Corresponding author: noorphkad@gmail.com
}

Received September 23, 2014; Revised October 05, 2014; Accepted October 12, 2014

\begin{abstract}
Objective: To assess the effects of administration of L-carnitine and coenzyme Q10 on glycemic control, lipid profile and lipoprotein (a) when added to pre-existing oral antidiabetic drugs in patients with type 2 diabetes. Methods: Fifty seven type 2 diabetic patients were randomly assigned into three groups for treatment with L-carnitine (1g daily), coenzyme Q10 (150mg daily), orcontinued on the same oral antidiabetic drugs (sulfonylurea and metformin) and considered as control group. All patients have been kept on the same medications throughout the study. Patients followed up after 8 weeks of treatment. Fasting blood glucose, glycated hemoglobin(HbA1c), total cholesterol, high-density lipoprotein cholesterol (HDL-c), low-density lipoprotein cholesterol (LDL-c) and lipoprotein (a) measured at baseline and after 8 weeks. Results: There is significant decrease in fasting blood glucose, total cholesterol, LDL-c and lipoprotein (a) in both L-carnitine group and coenzyme Q10 group after 8 weeks of treatment. In addition $\mathrm{HbA1c} \%$ significantly decreased in coenzyme Q10 group compared to control group. There were no significant differences in HDL-c in all three groups. Conclusion: Supplementation with L-carnitineor coenzyme Q10 improve glycemic control in type 2 diabetic subjects when added to conventional antidiabetic medications and could help in reducing the risk of cardiovascular complications by reduction of LDL-cholesterol and lipoprotein (a).
\end{abstract}

Keywords: L-carnitine, Coenzyme Q10, type 2 diabetes, glycemic control, lipid profile, lipoprotein (a)

Cite This Article: Noor Kadhim Mohammed-Jawad, May Al- Sabbagh, and Kaiss A. AL-Jezaeri, "Role of Lcarnitine and Coenzyme Q10 as Adjuvant Therapy in Patients with Type 2 Diabetes Mellitus.” American Journal of Pharmacological Sciences, vol. 2, no. 5 (2014): 82-86. doi: 10.12691/ajps-2-5-2.

\section{Introduction}

Diabetes is a group of metabolic diseases characterized by hyperglycemia resulting from defects in insulin secretion, insulin action, or both [1]. Diabetes mellitus is a global chronic metabolic disorder that represent one of the most challenging health problems inthe 21st century. People with diabetes have an increased risk of developing a number of serious health problems. Consistently high blood glucose levels can lead to serious diseases affecting the heart and blood vessels, eyes, kidneys, nerves and teeth [2].

The prevalence of diabetes has been increasing steadily all over the world due to the rapid urbanization, nutrition transition, and increasingly sedentary lifestyles [3]. In 2013, 382 million people had diabetes; this number is expected to rise to 592 million by 2035. Most people with diabetes live in low- and middle-income countries [4]. Type 2 diabetes comprises $90 \%$ of people with diabetes around the world [1]. In type 2 diabetes mellitus there is a significant role of insulin resistance, oxidative stress, and dyslipidemia in pathogeneses of disease and its complication [5]. Chronic complications are the major outcome of type 2 diabetes mellitus progress, which reduce the quality of life of patients, add heavy burdens to the health care system, and increase diabetic mortality [6]. Therefore there is a tremendous need for new management strategies which are safe and effective alone or in combination with existing drugs either for better control of the disease and/or prevent or delay its complications.

L-carnitine (1-3-hydroxy-4- $N, N$, $N$-tri-methylaminobutyrate) is a conditionally essential nutrient that plays a vital role in energy production and fatty acid metabolism.Carnitine is probably present in all animal species and in numerous micro-organisms and plants. In man L-carnitine synthesized from essential amino acid lysine [7]. L-carnitine has been recognized as a nutritional supplement in cardiovascular disease and there is increasing evidence that carnitine supplementation may be beneficial in treating obesity, improving glucose intolerance and total energy expenditure [8]. Food drug administration considered Lcarnitine as a drug to treat the primary and secondary carnitine deficiency [9].

Coenzyme Q10 (2, 3dimethoxy-5methyl-6-decaprenyl benzoquinone) is fat-soluble vitamin-likequinine commonly known as ubiquinone [10]. Coenzyme Q10 (Q10) is vital for the proper transfer of electrons and adenosine triphosphate production within the mitochondrial 
oxidative respiratory chain.Additionally, coenzyme Q10 has demonstrated activity in preventing lipid peroxidation and an indirect stabilizer of calcium channels to decrease calcium overload [11].Coenzyme Q10 extensively studied as a critical adjuvant therapy for patients with cardiac diseases due to its beneficial effects on cellular bioenergetics, regulation of cell membrane channels and antioxidant effect [12]. However there is few studies with a conflicting results about its possible effects in type 2 diabetes patients.

It's well known that diabetes mellitus carriesa high risk of cardiovascular complications, which remain themain cause of mortality in this population [13]. This study aimed to evaluate the proposed beneficial effects of short course (8 weeks) supplement of L-carnitine and coenzyme Q10 onglycemic control, total cholesterol, high density lipoprotein-cholesterol(HDL-c), low density lipoproteincholesterol(LDL-c) and lipoprotein (a)[Lp (a)] level which considered as a major risk factors for cardiovascular complications in patients with type 2 diabetes.

\section{Subjects and Methods}

\subsection{Study Design}

The present study was an 8-week randomized controlled single center clinical trial conducted at Specialized Center for Endocrinology and Diabetes, AlMawane General Hospital, Basra, Iraq carried out over 8 months from December 2013 till July 2014 at Specialized Center for Endocrinology and Diabetes, Al-Mawane General Hospital. Type 2 diabetes patients who treated with oral antidiabetic drugs (metformin and sulfonylurea)were randomly assigned into three groups to receive L-carnitine $1000 \mathrm{mg}$ tablet once daily,Coenzyme Q10 75mg soft gel twice daily, or continued on the same oral antidiabetic drugs and considered as control group. Lcarnitine was bought from Ultimate Nutrition Company, USA. Whereas Coenzyme Q10 was bought from Vitane's Nature Company, USA. Patients were evaluated at baseline and at week 8 .

Informed consent was obtained from all participants and an ethical approval was obtained from Ethical Committee of College of Pharmacy / Baghdad University.

\subsection{Sample Selection}

Eligible patients were previously diagnosed with type 2 diabetes mellitus according to the American Diabetes Association (ADA)Criteria. All the patients should be on the same oral antidiabetic drugs (sulfonylurea and metformin) for at least 3 consecutive months before the study and stabilized on same treatment throughout the study. Exclusion criteria include diabetic patients on insulin therapy, renalimpairment, hepatic impairment, and patients with co-existent thyroid disorders, autoimmune or chronic inflammatory disease.

\subsection{Laboratory Assessment}

Blood sample was taken at baseline and after 8 weeks for analysis of FBG, HbA1c, TC, LDL-c, HDL-c, and Lp (a). Laboratory analysis was done by specialized laboratory researchers who did not participate in this study. FBG, TC, LDL-c and HDL-c analyzed byclinical chemistry analyzer (Biolyzer 300, Germany). Lp (a) was measured by latexenhanced turbidimetic method using Lipoprotein (a) kit (Human, Germany). HbA1c measured by HBLC-based D10 Dual Program (Bio-Rad D-10, USA).

\subsection{Statistical Analysis}

Statistical analyses were performed with Statistical Package for Social Sciences (SPSS Inc., Chicago, IL, USA) version 19 for windows. Continuous variables were presented as mean \pm standard deviation (SD) and discrete variables were presented as numbers and frequencies. Chi square test for independence was used to test the significance of association between discrete variables. Continuous variables were checked by Q-Q plots and Shapiro Wilk test. ANOVA test was used to test the significance of difference in the mean of 3 independent samples in normally distributed continuous variables. Theend values of each continuous variable were also compared with the baseline values of it by using paired sample $t$-test for normally distributed variables while Wilcoxon rank test for abnormally distributed variables. The findings with $P$ values $<0.05$ were considered significant.

\section{Results}

\subsection{Patients}

Of seventy five patients presented to the study only fifty seven patients (26 male and 31 female) were complete the study. Their age were 51.07 \pm 7.27 years (as mean \pm SD) and duration of diabetes was $4.72 \pm 3.20$ years. There were no apparent differences between the three groups with respect to demographic data (Table 1).

Table 1. Patients characteristic at base line

\begin{tabular}{|c|c|c|c|c|}
\hline Characteristics & l-carnitinegroup & CoQ10 group & Control group & $\mathrm{p}$-value \\
\hline Age in years & $52.35 \pm 6.97$ & $49.37 \pm 6.65$ & $51.63 \pm 8.13$ & NS \\
\hline No. of patients & 19 & 19 & 19 & -------- \\
\hline $\begin{array}{l}\text { Male: Female } \\
\text { No. (\%) }\end{array}$ & $\begin{array}{l}8: 11 \\
(41 \%)\end{array}$ & $\begin{array}{l}10: 9 \\
(52 \%)\end{array}$ & $\begin{array}{l}8: 11 \\
(42 \%)\end{array}$ & NS \\
\hline BMI* & $29.47 \pm 3.8$ & $28.15 \pm 4.08$ & $29.5 \pm 4.29$ & NS \\
\hline Diabetes Duration( years) & $5.53 \pm 2.96$ & $4.76 \pm 3.98$ & $3.97 \pm 2.45$ & NS \\
\hline BaselineHbA1c\% & $9.29 \pm 2.15$ & $8.23 \pm 2.14$ & $8.53 \pm 1.94$ & NS \\
\hline
\end{tabular}

Data expressed as mean $\pm \mathrm{SD}$, ${ }^{\mathrm{BMI}}=$ body mass index, which is calculated according to the following formula: BMI= weight (kilogram)/hight2 (meter), NS= non-significant $(\mathrm{p}>0.05)$. 


\subsection{Fasting Blood Glucose and Glycated Hemoglobin}

Fasting blood glucose significantly $(\mathrm{p}<0.05)$ decrease in L-carnitine group compared to the baseline value, however no significant effect observed on $\mathrm{HbA} 1 \mathrm{c} \%$ after 8 weeks of treatment.

In Q10 group both FBG and HbA1c were significantly $(p<0.05)$ decrease compare to control group (Table 2$)$.

\subsection{Lipid Profile and Lipoprotein (a)}

Total cholesterol and LDL-c significantly decreased in both L-carnitine group and Q10 group compare to baseline value and non-significantly affected in control group. No significant effect on HDL-c was observed in all three groups.

Lipoprotein $(a)$ were significantly $(\mathrm{p}<0.05)$ decrease in L-carnitine group and Q10 group after 8 weeks of treatment compared to baseline valves and no statistical significant difference observed in control group (Table 2).

Table 2. Changes from baseline and after 8 weeks in laboratory parameters for patients who completed the study

\begin{tabular}{|c|c|c|c|}
\hline Parameter & $\begin{array}{l}\text { L-carnitine } \\
\text { (19) }\end{array}$ & $\begin{array}{l}\text { Coenzyme Q10 } \\
\text { (19) }\end{array}$ & $\begin{array}{l}\text { Control } \\
\text { (19) }\end{array}$ \\
\hline $\begin{array}{l}\text { FBG(mg/dl) } \\
\text { Before intervention } \\
\text { After intervention } \\
\% \text { of change }\end{array}$ & $\begin{array}{l}237.73 \pm 61.21 \\
196.4 \pm 77.62^{*} \\
-17.36 \% * * \\
\end{array}$ & $\begin{array}{l}198.12 \pm 61.54 \\
158.46 \pm 47.1^{*} \\
-20.02 \% * *\end{array}$ & $\begin{array}{l}182.25 \pm 46.9 \\
191.89 \pm 49.57 \\
5.28 \%\end{array}$ \\
\hline $\begin{array}{l}\text { HbA1c (\%) } \\
\text { Before intervention } \\
\text { After intervention } \\
\% \text { of change }\end{array}$ & $\begin{array}{l}9.26 \pm 2.06 \\
8.66 \pm 1.87 \\
-6.54 \%\end{array}$ & $\begin{array}{l}8.4 \pm 2.07 \\
7.3 \pm 1.65 * \\
-13.08 \% * * \\
\end{array}$ & $\begin{array}{l}8.1 \pm 1.61 \\
8.5 \pm 1.59 \\
5.01 \% \\
\end{array}$ \\
\hline $\begin{array}{l}\text { TC(mg/dl) } \\
\text { Before intervention } \\
\text { After intervention } \\
\% \text { of change }\end{array}$ & $\begin{array}{l}203.06 \pm 39.26 \\
184.5 \pm 33.2^{*} \\
-9.14 \%\end{array}$ & $\begin{array}{l}210.12 \pm 75.84 \\
180.9 \pm 48.4^{*} \\
-13.89 \%\end{array}$ & $\begin{array}{l}187.39 \pm 49.8 \\
198.82 \pm 49.4 \\
0.003 \%\end{array}$ \\
\hline $\begin{array}{l}\text { LDL(mg/dl) } \\
\text { Before intervention } \\
\text { After intervention } \\
\% \text { of change }\end{array}$ & $\begin{array}{l}110.15 \pm 16.65 \\
101 \pm 16.03 * \\
-8.3 \%\end{array}$ & $\begin{array}{l}131.87 \pm 42.05 \\
108.92 \pm 33.21^{*} \\
-15.57 \%\end{array}$ & $\begin{array}{l}100.33 \pm 28.28 \\
115.11 \pm 30.12 \\
13.81 \%\end{array}$ \\
\hline $\begin{array}{l}\text { HDL(mg/dl) } \\
\text { Before intervention } \\
\text { After intervention } \\
\% \text { of change }\end{array}$ & $\begin{array}{l}41.78 \pm 8.25 \\
42.24 \pm 6.15 \\
1.101 \%\end{array}$ & $\begin{array}{l}39.66 \pm 6.74 \\
39.03 \pm 7.49 \\
-1.57 \%\end{array}$ & $\begin{array}{l}41.56 \pm 7.96 \\
37.7 \pm 6.52 \\
-9.28 \%\end{array}$ \\
\hline $\begin{array}{l}\text { Lp (a) (mg/dl) } \\
\text { Before intervention } \\
\text { After intervention } \\
\% \text { of change }\end{array}$ & $\begin{array}{l}41.73 \pm 18.13 \\
25.03 \pm 9.47 * \\
-37.51 \%\end{array}$ & $\begin{array}{l}39.92 \pm 22.31 \\
27.16 \pm 7.27 * \\
-28.59 \%\end{array}$ & $\begin{array}{l}35.46 \pm 26.84 \\
36.92 \pm 25.55 \\
3.85 \%\end{array}$ \\
\hline
\end{tabular}

Data represented as mean \pm SD for baseline and end of study values;* significantly different when compared to pre-treatment level within the same group $(P<0.05)$;** significantly different when compared to control group $(\mathrm{p}<0.05)$.

\section{Discussion}

Diabetes mellitus is most common chronic disease worldwide. It's a growing cause of disability and premature death, mainly through cardiovascular disease [14]. Therefore it's requiring continuous medical care with multifactorial risk reduction strategies in addition to glycemic control to lower the risk of macro- and microvascular complications [15]. Carnitine covers important role in lipid and glucose metabolismas well as assisting in fuel sensing [16]. In addition Q10 play akey role in mitochondrial bioenergetics and plasma antioxidant effect and shown to improve lipid profile in serum, liver and muscle tissue in diabetic rat [17].This work aim to evaluate the effect of L-carnitine (1g/day) or coenzyme Q10 (75mg bid)on some metabolic parameters in type 2 diabetes when added to oral antidiabetic drugs (metformin and sulfonylurea).

The present study shows that L-carnitine administration over the period of 8 weeks significantly reduce FBG without significant reduction in $\mathrm{HbA} 1 \mathrm{c} \%$ compared to control group. This result is in agreement with Hadadinezhad et al., who find significant reduction in FBS but no significant effect on HbA1c and 2-hour post prandial blood glucose and c-peptide level [18]. It's possible that improvement in glucose metabolism insufficient to reach changes in glycated hemoglobin. However in Rahbaret al. study, L-carnitine shows a significant decrease in bothFBG and HbA1c [19]. The lack of effect on HbA1c in this study may attributed to short timeframe (8 weeks) of the study and lower dose of l-carnitine (1g/day) compared with Rahbar et al., who administer 3g/day of L-carnitine for 12 weeks. Abdelaleem et al. suggested that L-carnitine stimulates pyruvate dehydrogenase complex activity and enhances nonoxidative glucose metabolism by increasing in the mitochondrial acetyl carnitine efflux in the absence of exogenous fatty acidsin isolated myocytes [20].

The study also show also showed that supplementation with coenzyme Q10 in a dose of $75 \mathrm{mg}$ bid in combination with oral antidiabetic drugs associated with a significant decrease in both FBG and HbA1c after 8 weeks of intervention. This study is consistent with Hodgson et al., who report an improvement of long-term glycemic control after 12 weeks course of Q10 administration [21]. Several mechanisms suggested to explain the effect of coQ10 on glycemic control. Mezawa et al indicated that ubiquinol, the reduced form of Q10,improves insulin production and/or insulin secretion probably through activating mitochondrial ATP production inpancreatic beta-cells [22] Moreover Q10 might reduce oxidative stress in mitochondria and this might improve insulin sensitivity and b-cell function [23]. The result of this study inconsistence with Eriksson et al study, in which no 
significant improvement in glycemic control observed in 23 type 2 diabetes who receive $200 \mathrm{mg} /$ day Q10 or placebo for 6 months [24]. This inconsistency may attributed to small sample size in Eriksson et al and the differences in population, characteristics of patients and baseline HbA1c.

Both l-carnitine and Q10 show significant decrease in TC, LDL-c and Lp (a) after 8 weeks of treatment and nonsignificant effect on HDL-c.

Carnitine covers an important role in lipid metabolism, acting as an obligatory cofactor for beta-oxidation of fatty acids by facilitating the transport of long-chain fatty acids across the mitochondrial membrane as acylcarnitine esters [16]. Thus carnitine may shift the liver metabolism from esterification and synthesis of triglycerides toward the formation of acetylcarnitines. This could decrease synthesis of triglycerides and VLDL cholesterol [25]. Malaguarnera et al. observe a significant decrease in LDL cholesterol, oxidized LDL cholesterol, and triglycerides after 12 week in the carnitine-treated hypercholesterolemic newly diagnosed type 2 diabetes patients [26].Effect of Lcarnitine on lipids in this study is consistence with Alipuor et alin part that he also report decrease in TC and LDL in type 2 diabetic women with low caloric diet after 8 weeks of L-carnitine administration [27].

Conversely Rahbar et al [19] and González-Ortizet al [28] show a non-significant effect of L-carnitineon TC and LDL-c in type2 diabetic patients. This inconsistency in result could possibly attributed to relatively higher dose of L-carnitine (3 g/day) that used in Rahbar et al [19] and González-Ortiz et al[28]while in current work $1 \mathrm{~g} /$ day of L-carnitine used.

In addition Lp (a) shown to be reduced by significantly by administration of $1 \mathrm{~g} /$ day of L-carnitine. This result confirm the result of Derosa et al [29] and Sirtori et al [30] who reported that $2 \mathrm{~g}$ /day of L-carnitine reduce plasma level of lipoprotein(a) in diabetic and non-diabetic patients respectively without clinically relevant adverse events.

Lipoprotein (a) appeared to be one of the most atherogenic lipoproteins and may be a potential risk factor for cardiovascular disease. It's composed of liver-derived apo(a) covalently bound to apoB, which is similar in lipid composition to apoB of LDL [31]. Lp(a) plasma level strictly under genetic control of and relatively refractory to both lifestyle and drug intervention [32]. Nicotinic acid derivative is the only lipid lowering drug that reduce $\mathrm{Lp}(\mathrm{a})$ levels by reduction in the circulating FFA inflow into hepatocyte [33], however its use limited by tolerability issues. L-carnitine plays an important role in the mitochondrial uptake of long-chain fatty acids by facilitating their transportation across the inner mitochondrial membrane to undergo $\beta$-oxidation and may reasonably reduce level fatty acid in flow for apo(a) production [34].

Coenzyme Q10 supplement leads to significant decrease in serum cholesterol, and LDL-cwithout significant effect on HDL-c. This result confirm the results of Mohammadi et al in which 64 type 2 diabetic patients receive either $200 \mathrm{mg}$ Q10 or placebo daily for 12 weeks [35]. Q10 is a lipid-soluble substance that act as an intercellular antioxidant, and its presence was then demonstrated in all cell membranes and in blood, both in high- and in low-density lipoproteins [36]. Therefore reduction in lipid levels may be due to inhibition of LDL-
C oxidation. Chew et al shows that Q10 have no effect on lipid profile [37]. In chew et al study participants were typically overweight with satisfactory glycemic and lipid control while Q10 appears to be effective in hypercholesterolemic condition [38]. Q10 also significantly reduce $L p$ (a) but the magnitude of the effect variable among subjects, with some patients show a marked decrease and others only marginal change occur. Shojaei et al show that coenzyme Q10 in a dose of $100 \mathrm{mg} / \mathrm{d}$ is associated with a significant decrease in serum lipoprotein(a) level in maintenance hemodialysis patients [39]. Cicero et al demonstrated that adding CoQ10 to fenofibrate could improve the drug's efficacy in hypertriglycermia patients not responding to fenofibrate alone [40]. Mechanisms by which Q10 can decrease serum Lp (a) not well known. Q 10 was recognized to have an effect on gene expression [41]. Supplementation with coenzyme Q10 can inhibit expression of lipoprotein (a) receptor and result in decreased serum lipoprotein (a) [42].

\section{Conclusion}

Administration of L-carnitine(1g/day) and coenzyme Q10 (75mg bid) improve glycemic control, reduce total cholesterol and LDL-cholesterol in patient with type 2 diabetes when added to pre-existing oral antidiabetic drugs. L-carnitine and coenzyme Q10 also reduce lipoprotein (a) and thus may provide a protective effect against cardiovascular disease in diabetic patients. Further larger and longer studies are warranted to confirm these findings.

\section{Acknowledgement}

The present work was abstracted from MSc theses submitted to the Department of Clinical Pharmacy, College of Pharmacy, and University of Baghdad. The authors gratefully thank Specialized Center for Endocrinology and Diabetes, Al-Mawane General Hospital\Basra for supporting the project.

\section{References}

[1] American Diabetes Association. Diagnosis and Classification of Diabetes Mellitus. Diabetes Care 2014; 37 (1): 81-90.

[2] Sicree R, Shaw j and Zimmet p. Diabetes and Impaired Glucose Tolerance. IDFDiabetes Atlas, fourth edition, 2009.

[3] Hu F. B. Globalization of Diabetes The role of diet, lifestyle, and genes. Diabetes care 2011; 34(6): 1249-1257.

[4] Guariguata L, Whiting D. R, Hambleton I, Beagley J, Linnenkamp U and Shaw J. E. Global estimates of diabetes prevalence for 2013 and projections for 2035. Diabetes research and clinical practice 2014; 103(2):137-149.

[5] Soumya D. Late Stage Complications of Diabetes and Insulin Resistance. Journal of Diabetes \& Metabolism 2011; 2(9).

[6] Liu, Z., Chaowei, F., Weibing, W. and Biao, X. "Rersearch prevalence of chronic complications of type 2 diabetes mellitus in outpatients-a crosssectional hospital based survey in urban China." Health Qual Life Outcomes 8 (2010): 62.

[7] Vaz F. M and Wanders R. Carnitine biosynthesis in mammals. Biochem. J. 2002; 361: 417-429.

[8] Flanagan J, Simmons P, Vehige J, Willcox Mand Garrett Q.Role of carnitine in disease. Nutrition \& Metabolism 2010; 7(30). 
[9] Dayanand C. D, Krishnamurthy N, Ashakiran S and Shashidhar K. N. Carnitine: a novel health factor-an overview. Int J Pharm Biomed Res 2011; 2(2): 79-89.

[10] Kapoor P and Kapoor A. K. Coenzyme Q10-A novel molecule. Journal, Indian Academy of Clinical Medicine 2013; 14(1): 37-45.

[11] Bonakdar R. A and Guarneri E. Co-enzym Q10. Complementary and alternative medicine 2005; 72(6):1065-70.

[12] Kumar A, Kaur H, Devi P and Mohan V. Role of coenzyme Q10 (CoQ10) in cardiac disease, hypertension and Meniere-like syndrome. Pharmacology \& therapeutics 2009; 124(3): 259-268.

[13] Ajjan R. Cardiovascular disease in diabetes: are we doing enough? Austin Journal of Endocrinology and Diabetes 2014; 1(2).

[14] Unachukwu C and Ofori S. Diabetes mellitus and cardiovascular risk. The Internet Journal of Endocrinology 2012; 7(1).

[15] American Diabetes Association. Standards of medical care in diabetes-2014. Diabetes Care 2014; 37(1): 14-80.

[16] Mingrone G. Carnitine in type 2 diabetes. Annals of the New York Academy of Sciences 2004; 1033(1): 99-107.

[17] Amin M. M, Asaad G. F, Salam R, El-Abhar H. S and Arbid M. S. Novel CoQ10 Antidiabetic Mechanisms Underlie Its Positive Effect: Modulation of Insulin and Adiponectine Receptors, Tyrosine Kinase, PI3K, Glucose Transporters, sRAGE and Visfatin in Insulin Resistant/Diabetic Rats.PloS one 2014; 9(2): e89169.

[18] Hadadinezhad S, Ghazaleh N and Razavi Z. Effects of l-carnitine on glycemic control and C-peptide levels in patients with type 2 diabetes mellitus. Turk Jem 2008; 12: 1-3.

[19] Rahbar A. R, Shakerhosseini R, Saadat N, Taleban F, Pordal A and Gollestan B. Effect of L-carnitine on plasma glycemic and lipidemic profile in patients with type II diabetes mellitus. European journal of clinical nutrition 2005; 59(4): 592-596.

[20] Abdel-aleem S, Sayed-Ahmed M, Nada M. A, Hendrickson S. C, St Louis J and Lowe J E. Stimulation of Non-oxidative Glucose Utilization byL-carnitine in Isolated Myocytes. Journal of molecular and cellular cardiology 1995; 27(11): 2465-2472.

[21] Hodgson J. M, Watts G. F, Playford D. A, Burke V and Croft K. D. Original Communication-Coenzyme Q10 improves blood pressure and glycaemic control: a controlled trial in subjects with type 2 diabetes. European Journal of Clinical Nutrition 2002; 56:11371142.

[22] Mezawa M, Takemoto M, Onishi S, Ishibashi R, Ishikawa T, Yamaga, M, et al. The reduced form of coenzyme Q10 improves glycemic control in patients with type 2 diabetes: an open label pilot study. Biofactors 2012; 38(6): 416-421.

[23] Anwar M, El-Moniem M. A, Megahed H. A, EL-Toukhy S. E, Mohammed N. A and Youness E. R. Effect of Coenzyme Q10 Supplementation on Markers of Oxidative Stress in Streptozotocin Induced Diabetic Rats. Journal of Applied Pharmaceutical Science 2014; 4(6):9-15.

[24] Eriksson J. G, Forsen T. J, Mortensen S. A and Rohde M. The effect of coenzyme Q10 administration on metabolic control in patients with type 2 diabetes mellitus. Biofactors 1999; 9(2-4): 315-318.

[25] Mynatt R. L. Carnitine and type 2 diabetes. Diabetes/metabolism research and reviews 2009; 25(S1): 45-49.

[26] Malaguarnera M, Vacante M, Avitabile T, Malaguarnera M, Cammalleri L and Motta M. L-Carnitine supplementation reduces oxidized LDL cholesterol in patients with diabetes. The American journal of clinical nutrition 2009; 89 (1), 71-76.

[27] Alipour B, Barzegar A, Panahi F, Safaeian A and Es. haghi M. Effect of L-Carnitine Supplementation on Metabolic Status in Obese Diabetic Women with Hypocaloric Diet. Health Scope 2014; 2(4): e14615.
[28] Gonzalez-Ortiz M, Hernandez-Gonzalez S. O, Hernandez-Salazar $\mathrm{E}$ and Martinez-Abundis E. Effect of oral L-carnitine administration on insulin sensitivity and lipid profile in type 2 diabetes mellitus patients. Annals of Nutrition and Metabolism 2008; 52(4): 335-338.

[29] Derosa G, Cicero A. F, Gaddi A, Mugellini A, Ciccarelli L and Fogari R. The effect of L-carnitine on plasma lipoprotein (a) levels in hypercholesterolemic patients with type 2 diabetes mellitus. Clinical therapeutics 2003; 25 (5):1429-1439.

[30] Sirtori C. R, Calabresi L, Ferrara S, Pazzucconi F, Bondioli A, Baldassarre D, et al. L-carnitine reduces plasma lipoprotein (a) levels in patients with hyper Lp (a). Nutrition, metabolism, and cardiovascular diseases: NMCD2000; 10(5): 247-251.

[31] Tsimikas S and Hall J. L. Lipoprotein (a) as a potential causal genetic risk factor of cardiovascular disease: a rationale for increased efforts to understand its pathophysiology and develop targeted therapies. Journal of the American College of Cardiology 2012; 60 (8): 716-721.

[32] Kostner K. Lipoprotein (a): Pathophysiology, Metabolism, Treatment Options. Austral - Asian Journal of Cancer 2007; 6 (3): 157-163.

[33] Kei A andElisaf M. S. Nicotinic acid: Do we know how it works after 55 years of clinical experience. World J Pharmacol 2012 June 9; 1(3): 50-54.

[34] Galvano F, Li Volti G, Malaguarnera M, Avitabile T, Antic T, Vacante $M$ and Malaguarnera $M$. Effects of simvastatin and carnitine versus simvastatin on lipoprotein (a) and apoprotein (a) in type 2 diabetes mellitus. Expert Opin. Pharmacother. 2009; 10(12); 1875-82.

[35] Mohammadi R. K, Hosseinzadeh-Attar M. J, Eshraghian M. R and Esteghamati A. Coenzyme Q10 supplementation on metabolic status of type 2 diabetic patients. 1st Tabriz International Life Science Conference and 12th Iran Biophysical Chemistry Conference 2013. Tabtiz University of medical sciences.

[36] Garrido-Maraver J, Cordero M. D, Oropesa-Ávila M, et al. Coenzyme Q10 Therapy. Molecular Syndromology 2014; 5(3-4): 187-197.

[37] Chew G. T, Watts G. F, Davis T. M,et al. Hemodynamic effects of fenofibrate and coenzyme Q10 in type 2 diabetic subjects with left ventricular diastolic dysfunction. Diabetes Care 2008; 31(8): 1502-1509.

[38] Al-Attar, A. M. (2010). Hypolipidemic effects of coenzyme Q10 in experimentally induced hypercholesterolemic model in female rats. American Journal of Pharmacology and Toxicology, 5(1), 1423.

[39] Shojaei M, Djalali M, Khatami M, Siassi F and Eshraghian M. Effects of carnitine and coenzyme Q10 on lipid profile and serum levels of lipoprotein (a) in maintenance hemodialysis patients on statin therapy. Iran J Kidney Dis 2011; 5(2): 114-118.

[40] Cicero A, Derosa G, Miconi A, et al. Possible role of ubiquinone in the treatment of massive hypertriglyceridemia resistant to PUFA and fibrates. Biomedicine \& pharmacotherapy 2005; 59(6): 312-317.

[41] Groneberg D. A, Kindermann B, Althammer M, et al. Coenzyme $\mathrm{Q}<$ sub $>10</$ sub $>$ affects expression of genes involved in cell signalling, metabolism and transport in human CaCo-2 cells. The international journal of biochemistry \& cell biology 2005; 37(6): 1208-1218.

[42] Singh R. B andNiaz M. A. Serum concentration of lipoprotein (a) decreases on treatment with hydrosoluble coenzyme Q10 in patients with coronary artery disease: discovery of a new role. International journal of cardiology 1999; 68(1): 23-29. 